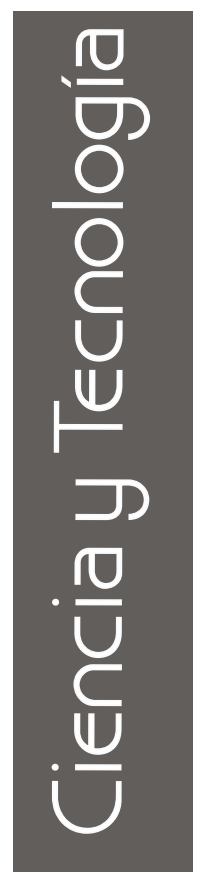

\title{
Mejora del Rendimiento Operativo y Financiero de las Cadenas de Suministro mediante el uso de las Herramientas de Colaboración basadas en Internet. Un Enfoque Sistémico
}

\author{
Oscar Rubiano Ovalle*
}

* Ph.D. Profesor Asistente - Escuela de Ingeniería Industrial y Estadística - Facultad de Ingeniería - Universidad del Valle Santiago de Cali.

E-mail:oscaruba@pino.univalle.edu.co
Fecha de recepción: Octubre 3 de 2002

Fecha de aprobación: Marzo 26 de 2003

\section{RESUMEN}

Este artículo evalúa el posible impacto de las nuevas tecnologías de la comunicación sobre el rendimiento operativo de una cadena de suministro (CS) genérica. Con el objeto de conceptualizar los procesos de integración que se pueden llevar a cabo en la cadena de suministro, se realizó un estudio de simulación. El estudio contiene un modelo construido para medir las mejoras en la gestión de la cadena de suministro, como una consecuencia de un mayor flujo de información compartido.

El modelo es de simulación con dinámica de sistemas y su estructura básica se obtuvo con 
base en el juego de la cerveza (Sterman 1984). Los resultados de este trabajo muestran órdenes de magnitud de las mejoras en términos de medidas operativas y financieras.

Palabras Claves: Cadenas de Suministro, Comercio en colaboración, Simulación, Dinámica de Sistemas.

\section{ABSTRACT}

This paper assesses the possible impact of the new communication technologies on the operative performance of a generic supply chain. A simulation study has been accomplished in order to conceptualize the integration process that could take place in the supply chain, and to measure improvements in supply chain speed and flexibility as a consequence of a faster and shared information flow.

The paper presents results for a system dynamics simulation model, whose basic structure was built from the beer game (Sterman 1984). The results show orders of magnitude of the improvements in terms of operational and financial metrics.

Keywords: Supply Chain Management, Commerce, Simulation, System Dynamics.

\section{INTRODUCCIÓN}

El concepto "gestión de la cadena de suministro" (acuñado en inglés como supply chain management, SCM), se usa en esta publicación haciendo alusión a los medios por los cuales las empresas abordan la creación, distribución y venta de sus productos (Poirier 1999). Esto incluye todos los esfuerzos cooperativos entre los miembros de la Cadena de Suministro (CS), para alcanzar un mayor conocimiento del mercado, mediante la tenencia de una información conjunta y más precisa del mercado, la investigación de productos el desarrollo y diseño de productos, y el análisis de valor del sistema total (Bowersox 1997, Cavinato 1992,
Mentzer 1993). Esta red de suministro puede convertirse en una ventaja competitiva sumamente poderosa para las organizaciones industriales; es evidente que en realidad, la competencia real ya no es compañía vs. compañía, sino CS vs. CS (Christopher 1992). Los avances en las tecnologías de la comunicación (con aplicaciones de bajos costos de acceso, además de los estándares emergentes para el intercambio de datos, como $X M L$ ), y la información (sistemas que hoy llamados ERP) contribuyen apropiadamente a la gestión de una CS; permiten que los sistemas "viajen" a través de las múltiples organizaciones y faciliten el flujo de información desde cualquier fuente hasta todas las firmas integrantes de la CS.

En la siguiente sección de este artículo se discute en primer lugar el concepto de integración de una CS y se revisan las contribuciones principales encontradas en este tema. En la sección 3 se discuten los tipos de información compartida y el uso de herramientas electrónicas de colaboración (e-collaboration tools) para tal fin dentro de una CS, así como las principales contribuciones en este aspecto. En la sección 4 se introduce un modelo básico para la representación de la CS y en la sección 5 , se especifica la manera como se formalizan los diferentes niveles de integración en el modelo. La sección 6 está dedicada al modelado del capital circulante y otras variables financieras de la CS, haciendo énfasis en las necesidades de fondos. En la sección 7 se discuten las salidas del modelo con la intención de validar el comportamiento de las variables del mismo y aplicarlo a un caso supuesto. Finalmente en la sección 8 se presentan las conclusiones sobre los resultados del modelo.

\section{REVISIÓN BIBLIOGRÁFICA SOBRE INTEGRACIÓN EN LA CADENA DE SUMINISTRO}

A pesar de la relevancia del tema de la integración dentro del CS, éste ha sido abordado 
ampliamente por relativamente pocos investigadores. Entre las contribuciones principales están las realizadas por Stevens (1989), Scott y Westbrok (1991), Towill et al. (1992), Cooper y Ellram (1993), Hewitt (1994) y Bowersox (1997).

Stevens (1989) presentó un modelo de integración con cuatro fases: integración básica, integración funcional interna, integración del suministro y la demanda de las propias compañías a lo largo de la cadena, y la integración total de la cadena de suministro. Hewitt (1994) extendió el modelo de Stevens a una quinta fase que se podría dedicar a una mejor gestión y rediseño de los procesos de negocio globales, buscando mejorar la eficiencia y la eficacia globales de tales procesos. Scott y Westbrook (1991) sugirieron un modelo de tres fases para obtener una cadena de suministro integrada: 1) la "fase de estudio", donde se analizan los lead times y los niveles de inventario para ver mejoras potenciales, 2) una "fase de posicionamiento", para identificar nuevas oportunidades que surgen como consecuencia de las actividades de colaboración entre los miembros de la cadena, y 3) una "fase de acción" para llevar a cabo los planes anteriores. Towill et al. (1992) presentaron un enfoque de integración, similar al de Stevens, y se basaron en principios de dirección de operaciones para reducir la amplificación de la señal de la demanda a lo largo de la cadena. Bowersox (1997) plantea la idea de dos tipos de integración: interna y externa, y sugiere que la creación de beneficios de tiempo y ubicación en la cadena, exige compartir la información permitiendo acuerdos comerciales convenientes con ese propósito, y también exige la existencia de un ambiente apropiado para las transacciones financieras.

\section{TIPOS DE FORMACIÓN COMPARTIDA Y HERRAMIENTAS DE COLABORACIÓN}

La información compartida podría ser acerca de los inventarios, las ventas, la previsión de la demanda, el estado de las órdenes, la planeación del producto, la logística, los programas de la producción, etc., y puede resumirse en tres tipos: información sobre el producto, información sobre la demanda de los clientes y las transacciones, e información sobre los inventarios.

Hoy en día organizaciones como UCCnet proveen algunos esfuerzos para el intercambio de información sobre el producto. Estas organizaciones van directamente a los proveedores para capturar los datos (a partir de sus propios formatos) y los traslada vía XML en un formato al cual los detallistas eslabonados puedan tener acceso.

La información sobre la demanda de los clientes y las transacciones sirve como fuente crítica de información acerca de los negocios futuros, y es usada directamente para la previsión de la demanda, los programas de producción, la planificación del transporte, etc. El recientemente desarrollado Collaborative Forecasting and Replenishment (CFAR), es un nuevo sistema interorganizacional que permite a los detallistas y fabricantes prever la demanda y programar conjuntamente la producción (Raghunathan 1999).

En la práctica, hay varias formas para implementar el sistema para compartir la información sobre los inventarios. EI CRP (Continuous Replenishment Programs) O Vendor-Managed Inventory (VMI) es una práctica empleada a menudo por dos firmas socias vecinas de la CS. En una relación típica CRP, el comprador comparte los datos de su inventario con el vendedor, y le invita a manejar sus inventarios dentro de unos lineamientos. El programa Wal-Mart's Retail Link (Gill and Abend 1997) y Apple-Fritz Supplier Hub (Lee and Whang 1998) son buenos ejemplos de información de los inventarios compartida. 
Mejora del Rendimiento Operativo y Financiero de las Cadenas de Suministro mediante el uso de Herramientas de Colaboración basadas en Internet. Un Enfoque Sistémico

\section{MODELO DE LA CADENA DE SUMINISTRO}

La notación usada para los flujos de información y de materiales es la siguiente:

\section{* Variables de estado y flujo de materiales:}

$P_{t}^{i}$ :Trabajo en proceso del nodo $i$ en el período $t$,

$Y_{t}^{i}$ :Inventario de materiales/productos terminados en el nodo $i$ (inventario a mano) en el período $t$,

$S_{t}^{i}$ :cantidad de unidades enviadas al siguiente nodo $i+1$ en el período $t$,

$O_{t}^{i}$ : unidades salientes del inventario de trabajo en proceso del nodo $i$ en el período $t$, tasa de procesamiento de unidades,

$I_{t}^{i}$ : unidades entrantes al inventario de trabajo en proceso del nodo $i$ en el período $t$,

\section{* Variables de estado y flujo de información, órdenes y entregas}

$\hat{\mu}_{t}^{i}$ : previsión de la demanda del nodo $i$ en el período $t$,

$O P_{t}^{i}$ :órdenes colocadas por el nodoi en el período $t$,

$D_{t}^{i+1}$ :órdenes recibidas en el nodo $i$ en el período $t$,

$B_{t}^{i}$ :órdenes pendientes de satisfacer por parte del nodo $i$ en el período $t$,

$D S_{t}^{i}$ :entregas deseadas del nodo $i$ al nodo $i+1$ en el período $t$,

$T Y_{t}^{i}$ :materiales/productos totales disponibles en el nodo $i$, en el período $t$,

$D O_{t}^{i}$ :cantidad de órdenes finalmente enviadas al nodo siguiente $i+1$ (equivalente a las unidades enviadas al siguiente nodo $S_{t}^{i}$ ) en el período $t$,

\section{* Parámetros del modelo}

$\hat{\mu}_{t}$ :previsión de la demanda en periodo $t$,

$L^{i}$ :tiempo de proceso (lead time), para que una unidad de material del trabajo en proceso llegue al inventario de materiales/productos terminados, en el nodo $i$,
$T D^{i}$ :Tiempo de entrega del nodo $i$ (igual a la unidad, los productos se entregan en el mismo período en que se reciben los pedidos),

$I D^{i}$ :tiempo para que la información sobre las órdenes colocadas por la etapa $i$ sea recibida y se procese en la etapa $i+1$,

$S S^{i}$ :tiempo deseado de permanencia de una unidad de material, como inventario a mano en el nodo $i$ ( política de cada nodo),

$\alpha^{i}$ :factor de suavización de la previsión de la demanda en el nodo $i$,

$\beta_{s}$ :coeficiente de ajuste fraccional del inventario a mano,

$\beta_{s L}$ :coeficiente de ajuste fraccional del inventario de trabajo en proceso.

En la tabla 1 se presentan las ecuaciones del modelo.

\begin{tabular}{|c|c|}
\hline Ecuación & Mo. \\
\hline $\begin{array}{c}\text { Cadena de Suministro Básica } \\
\text { (asumida como la Mo Integrada, MI) }\end{array}$ & \\
\hline 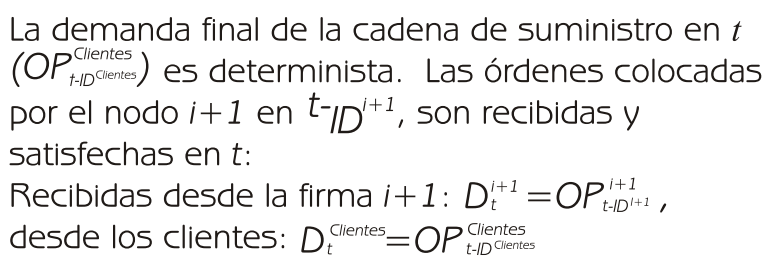 & {$[1]$} \\
\hline $\begin{array}{l}\text { Las órdenes a entregar deseadas, no incluyen } \\
\text { órdenes pendientes (estructuras de base): Desde } \\
\text { el nodo } i: D S_{t}^{\prime}=D_{t}^{\prime+1} \text {, desde el nodo } n: D S_{t}^{n}=D_{t}^{\text {clentes }}\end{array}$ & {$[2]$} \\
\hline $\begin{array}{l}\text { El suministro de órdenes } S_{t}^{i} \text {, al nodo siguiente/ } \\
\text { clientes finales, puede afectarse por limitaciones } \\
\text { del inventario, generándose la variable unidades } \\
\text { totales disponibles } T Y_{t}^{\prime} \text { : } \\
\text { desde la firma } i: S_{t}^{\prime}=\text { MIM }\left(T Y_{t}^{i}, D S_{t}^{i}\right)\end{array}$ & [3] \\
\hline $\begin{array}{l}\text { Las unidades totales disponibles del período } t \\
\text { incluyen las recepciones de materiales: } \\
T Y_{t}^{\prime}=Y_{t}^{\prime}+O_{t}^{\prime}\end{array}$ & [4] \\
\hline $\begin{array}{l}\text { Órdenes pendientes } B_{t}^{\prime} \text { (suponiendo que las condi- } \\
\text { ciones iniciales se conocen): } B_{t}^{\prime}=B_{t-1}^{\prime}+D_{t}^{i+1}-D O_{t}^{\prime}\end{array}$ & [5] \\
\hline
\end{tabular}




\begin{tabular}{|c|c|}
\hline Ecuación & 10. \\
\hline $\begin{array}{c}\text { Cadena de Suministro Básica } \\
\text { (asumida como la Mo Integrada, MI) }\end{array}$ & \\
\hline $\begin{array}{l}\text { Saldo físico del inventario a mano } Y_{t}^{i} \text { (suponiendo } \\
\text { condiciones iniciales conocidas): } \\
Y_{t}^{\prime}=Y_{t-1}^{\prime}+O_{t}^{\prime}-S_{t}^{\prime}\end{array}$ & ] \\
\hline $\begin{array}{l}\text { Registro de la información relativa a las órdenes } \\
\text { finalmente enviadas: } D O_{t}^{\prime}=S_{t}^{\prime}\end{array}$ & \\
\hline $\begin{array}{l}\text { Tasa de "terminación" del trabajo en proceso: } \\
O_{t}^{\prime}=l_{t-L^{\prime}}^{\prime}\end{array}$ & \\
\hline $\begin{array}{l}\text { Entrada al inventario de trabajo en proceso al } \\
\text { nodo } i \text { en } t: I_{t}^{\prime}=S_{t}^{1-1}\end{array}$ & [9] \\
\hline $\begin{array}{l}\text { Trabajo en proceso (asumiendo que se conocen } \\
\text { sus condiciones iniciales): } \\
P_{t}^{\prime}=P_{t-1}^{\prime}+l_{t}^{\prime}-O_{t}^{\prime}\end{array}$ & \\
\hline $\begin{array}{l}\text { Previsión de la demanda para el nodo i, método } \\
\text { de suavización exponencial: }\end{array}$ & \\
\hline $\begin{array}{l}\hat{\mu}_{t}^{i}=\alpha^{i} D_{t-1}^{i+1}+\left(1-\alpha^{i}\right) \hat{\mu}_{t-1}^{i}, 0<\alpha^{i} \leq 1, \forall i \\
\text { (Makridakis et al. 1998) }\end{array}$ & \\
\hline $\begin{array}{l}\text { Órdenes (asumiendo no integración en la } \\
\text { cadena de suministro): }\end{array}$ & \\
\hline$O P_{t}^{i}=\operatorname{Max}\left(\hat{\mu}_{t}^{i}+\hat{\mu}_{t}^{i}\left(\beta_{S} S S_{t}^{i}+\beta_{S L} L^{i}\right)-\left(\beta_{S} Y_{t}^{i}+\beta_{S L} P_{t}^{i}\right), 0\right)$ & \\
\hline $\begin{array}{l}\text { Órdenes: en la forma de la heurística de anclaje } \\
\text { y ajuste } 1 \text { (Tversky y Kahneman, 1974) con los } \\
\text { ajustes fraccionales de los inventarios y del } \\
\text { trabajo en proceso. La previsión de la demanda } \\
\text { se identifica como el término inercial, y los otros } \\
\text { dos términos son los de ajuste. Incluye la no } \\
\text { negatividad de los pedidos (Sterman, 1989): }\end{array}$ & \\
\hline$O P_{t}^{i}=\operatorname{Max}\left(\hat{\mu}_{t}^{i}+\beta_{S}\left(\hat{\mu}_{t}^{i} S S_{t}^{i}-Y_{t}^{i}\right)+\beta_{S L}\left(\hat{\mu}_{t}^{i} L^{i}-P_{t}^{i}\right), 0\right)$ & \\
\hline $\begin{array}{l}\text { Modificaciones para obtener la Cadena de } \\
\text { suministro Parcialmente Integrada (PI) }\end{array}$ & \\
\hline $\begin{array}{l}\text { Órdenes (descuenta las unidades pedidas con } \\
\text { anterioridad y aún no recibidas): }\end{array}$ & \\
\hline$O P_{t}^{i}=\operatorname{Max}\left(\hat{\mu}_{t}^{i}+\hat{\mu}_{t}^{i}\left(s S_{t}^{i}+L^{i}\right)-\left(P_{t}^{i}+Y_{t}^{i}\right)-B_{t-1}^{i-1}+i b_{t}^{i}, O\right)$ & \\
\hline $\begin{array}{l}\text { Información sobre inventarios de los nodos } \\
\text { siguientes, en el banco de información en } t \text { : } \\
i b_{t}^{i}=\hat{\mu}_{t}^{i}\left(\mathrm{SS}_{t}^{i+1}+L^{i+1}\right)-\left(P_{t}^{i+1}+Y_{t}^{i+1}\right)+i b_{t}^{i+1}\end{array}$ & \\
\hline $\begin{array}{l}\text { Entregas deseadas (incluye las órdenes recibidas } \\
\text { con anterioridad y aún no satisfechas): } \\
D S_{t}^{\prime}=B_{t-1}^{\prime}+D_{t}^{\prime+1}\end{array}$ & \\
\hline
\end{tabular}

\footnotetext{
${ }^{1}$ Traducción realizada del nombre original en inglés: anchoring and adjustment heuristic.
}

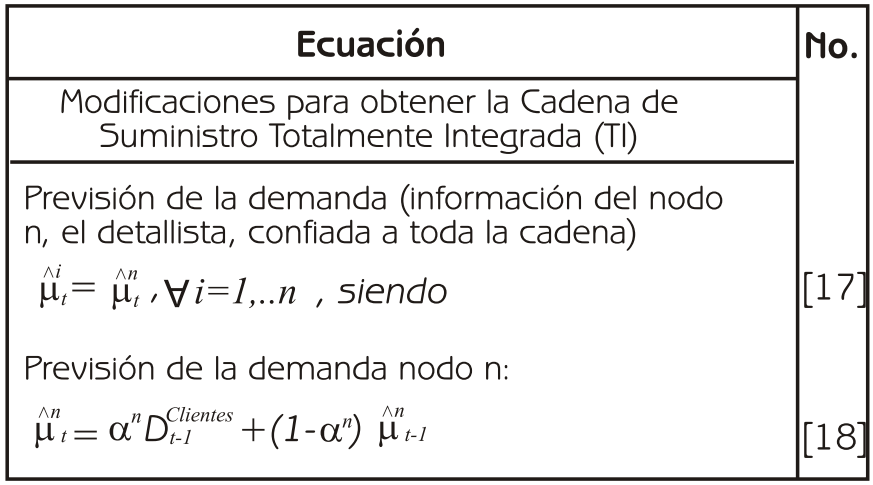

Tabla 1. Ecuaciones del modelo de Cadena de Suministros firma i

\section{MODELADO DE LOS NIVELES DE INTEGRACIÓN DE LA CADENA DE SUMINISTRO}

\section{Escenarios:}

En esta publicación se propone una secuencia (figura 1) para mejorar el rendimiento de la cadena de suministro, mediante un aumento gradual de la colaboración o integración.

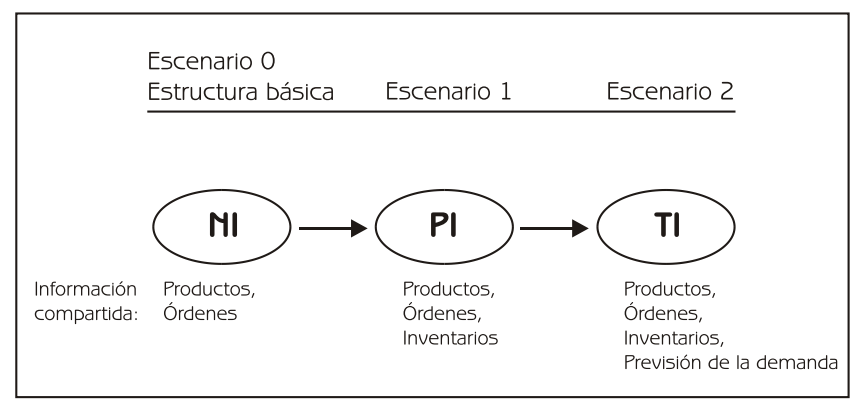

Figura 1. Pasos para la extensión de la colaboración en la cadena de suministro

\section{1.- Escenario 0: Cadena de Suministro no Integrada}

Cuando la estructura de la cadena de suministro es no integrada, las entregas de productos se definen de acuerdo a la ecuación [3] dada para la estructura base; la tasa de órdenes a colocar al nodo anterior, depende de la previsión de la demanda local y de los inventarios locales, de acuerdo con las ecuaciones dadas en [11] y en [12]. Cada nodo realiza su propia previsión de la demanda y ordena en concordancia con ello. 
Mejora del Rendimiento Operativo y Financiero de las Cadenas de Suministro mediante el uso de Herramientas de Colaboración basadas en Internet. Un Enfoque Sistémico

5.2.- Escenario 1: Cadena de Suministro Parcialmente Integrada

En la estructura PI los nodos conocen los niveles de inventarios de sus clientes directos e indirectos. Aquí también aplica la ecuación [11] para estimar la previsión de la demanda, pero las órdenes se calculan con la ecuación [14] que reemplaza a la [12].

\section{3.- Escenario 2: Cadena de Suministro Totalmente Integrada}

Al compartir también la información global sobre los clientes finales, la previsión de la demanda de todos los nodos se hace equivalente a la previsión de la demanda del último nodo, el detallista. En consecuencia, la ecuación [11] se reemplaza por la ecuación [17]. Las órdenes se calculan de la forma expresada en la ecuación [12].

\section{MODELADO DE LOS FLUJOS FINANCIEROS}

En este artículo se presenta sólo la relación entre las principales variables financieras relacionadas con la liquidez para cada nodo, cuya notación es la siguiente:

$C R_{t}^{i} \quad$ :necesidades de fondos (capital de trabajo) del nodo $i$ en el tiempo $t$,

$I C R_{t}^{i} \quad$ :necesidades de fondos para inventarios del nodo $i$ al tiempo $t$, incluye las necesidades de efectivo para financiar los inventarios finales y de trabajo en proceso,

$R_{t}^{i} \quad$ :cuentas por cobrar del nodo $i$ en el tiempo, $\mathrm{t}$.

$P y_{t}^{i} \quad$ :cuentas por pagar del nodo $i$ en el tiempo $t$,

$\mathrm{Pm}_{t}^{i} \quad$ :Precio de una unidad de producto enviada desde el nodo $i$ en $t$

$P w i p_{t}^{i}$ :valor medio de una unidad de trabajo en proceso en el nodo $i$ en $t$,

$m r_{t}^{i} \quad$ :margen de utilidad por producto en el nodo $i$ en el tiempo $t$,

$d s o(i)$ :período de cobro del nodo $i$,

CumP $_{t}^{i}$ : beneficios acumulados del nodo i, en t.
Las principales relaciones entre las variables es la siguiente:

$$
\begin{aligned}
& C R_{t}^{i}=I C R_{t}^{i}+R_{t}^{i}-P y_{t}^{i}-C u m P_{t}^{i} \\
& P y_{t}^{i}=P y_{t-1}^{i}+S_{t}^{i-1} P m_{t}^{i-1}-S_{t-d s o(i-1)}^{i-1} P m_{t-d s o(i-1)}^{i-1} \\
& R_{t}^{i}=R_{t-1}^{i}+S_{t}^{i} P m_{t}^{i}-S_{t-d s o(i)}^{i} P m_{t-d s o(i)}^{i} \\
& I C R_{t}^{i}=P_{t}^{i} P w i p_{t}^{i}+Y_{t}^{i}\left(P m_{t}^{i}-m r_{t}^{i}\right) \\
& C u m P_{t}^{i}=\sum_{k=1}^{k=t} S_{k}^{i} m r_{k}^{i}
\end{aligned}
$$

\section{APLICACIÓN A UN CASO SUPUESTO Y ANÁLISIS DE LOS RESULTADOS}

Para la validación de los patrones de comportamiento de las principales variables del modelo, así como para la aplicación y evaluación, se construyó el siguiente caso: (a) cuatro nodos en línea (Figura 2), a partir de los cuales, la cadena entrega productos al cliente final, (b) se asigna a los parámetros operativos (lead times, tiempos de retrasos, etc.), los valores asignados por Sterman (1984) en la realización del juego de la cerveza (Tabla 2).

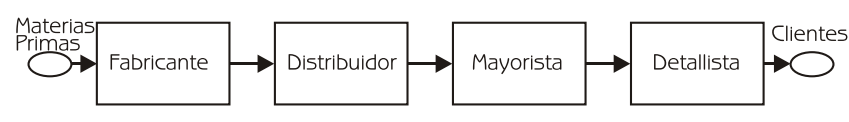

Figura 2. Cadena de suministro seleccionada

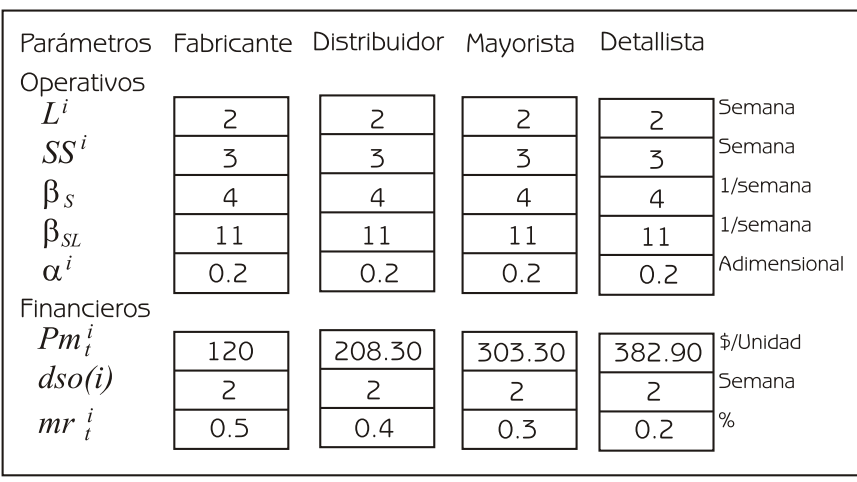

Tabla 2. Parámetros de simulación 
La demanda de los clientes es supuesta en 4 unidades/semana - constante, hasta cuando se da un pulso en la señal de la demanda en la semana número 5, aumentando su valor a 8 unidades/semana. El tiempo de demora en la llegada y procesamiento de las órdenes (ID') es una semana en todas las firmas. Los stocks de seguridad se estiman para alcanzar niveles de servicio (\% de órdenes entregados a tiempo) cerca del $95 \%$ en cada nodo.

La tabla 3 muestra los resultados sobre las órdenes colocadas por cada nodo, observándose por un lado, una reducción significativa en la oscilación (y la amplificación) de la demanda para las cadenas de suministro parcial y totalmente integradas. Esta Tabla se complementa con la figura 3 , en la cual se observa que para las órdenes colocadas, por ejemplo por el fabricante en la estructura no integrada, el retraso en la información de los pedidos colocados conduce a una tardía respuesta al cambio en la tasa de producción/aprovisionamiento.

\begin{tabular}{|c|c|c|c|c|c|}
\hline & & Min. & Máx. & Desv. Estad. & Media \\
\hline $\begin{array}{l}\text { Mo } \\
\text { Integrada }\end{array}$ & $\begin{array}{l}\text { Fabricante } \\
\text { Distribuidor } \\
\text { Mayorista } \\
\text { Detallista }\end{array}$ & $\begin{array}{l}0 \\
0 \\
1 \\
4\end{array}$ & $\begin{array}{l}34 \\
27 \\
20 \\
14\end{array}$ & $\begin{array}{c}11 \\
9 \\
6 \\
3\end{array}$ & $\begin{array}{l}14 \\
11 \\
9 \\
8\end{array}$ \\
\hline $\begin{array}{l}\text { Parcialmente } \\
\text { Integrada }\end{array}$ & $\begin{array}{l}\text { Fabricante } \\
\text { Distribuidor } \\
\text { Mayorista } \\
\text { Detallista }\end{array}$ & $\begin{array}{l}0 \\
2 \\
4 \\
4\end{array}$ & $\begin{array}{l}34 \\
21 \\
15 \\
11\end{array}$ & $\begin{array}{l}10 \\
5 \\
3 \\
2\end{array}$ & $\begin{array}{l}9 \\
9 \\
8 \\
8\end{array}$ \\
\hline $\begin{array}{l}\text { Totalmente } \\
\text { Integrada }\end{array}$ & $\begin{array}{l}\text { Fabricante } \\
\text { Distribuidor } \\
\text { Mayorista } \\
\text { Detallista }\end{array}$ & $\begin{array}{l}4 \\
4 \\
4 \\
4\end{array}$ & $\begin{array}{l}20 \\
15 \\
13 \\
10\end{array}$ & $\begin{array}{l}4 \\
3 \\
3 \\
2\end{array}$ & $\begin{array}{l}9 \\
8 \\
7 \\
7\end{array}$ \\
\hline
\end{tabular}

Tabla 3. Órdenes colocadas por nodo

En términos del inventario total (trabajo en proceso + partes/productos terminados) por firma, la figura 4 revela que una ventaja de la colaboración entre las firmas, es la obtención de

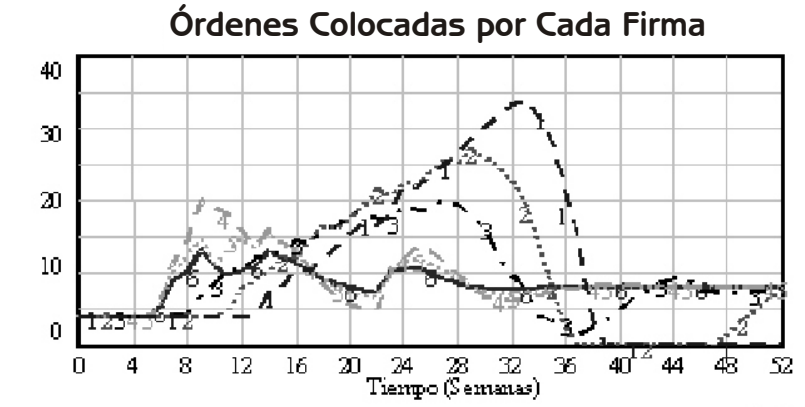

Fabricante CS MI $\left\ulcorner--_{1}-\neg--\neg_{-}--_{-}-\neg_{-}-\square\right.$ Unids./Sem

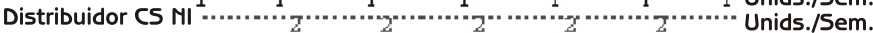
Mayorista CS MI $\rightarrow \cdot-3 \cdot-5 \cdot-5 \cdot-3 \cdot-3 \cdot-3$ Unids./Sem. Fabricante CS TI $-\frac{\pi}{4}-\frac{\pi}{4}-\frac{\pi}{4}-\frac{\pi}{4}-\frac{\pi}{4}-\frac{\pi}{4}-$ Unids./Sem.

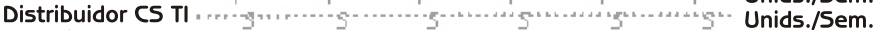
Mayorista CS TI

Figura 3. Resultados sobre la oscilación de la demanda

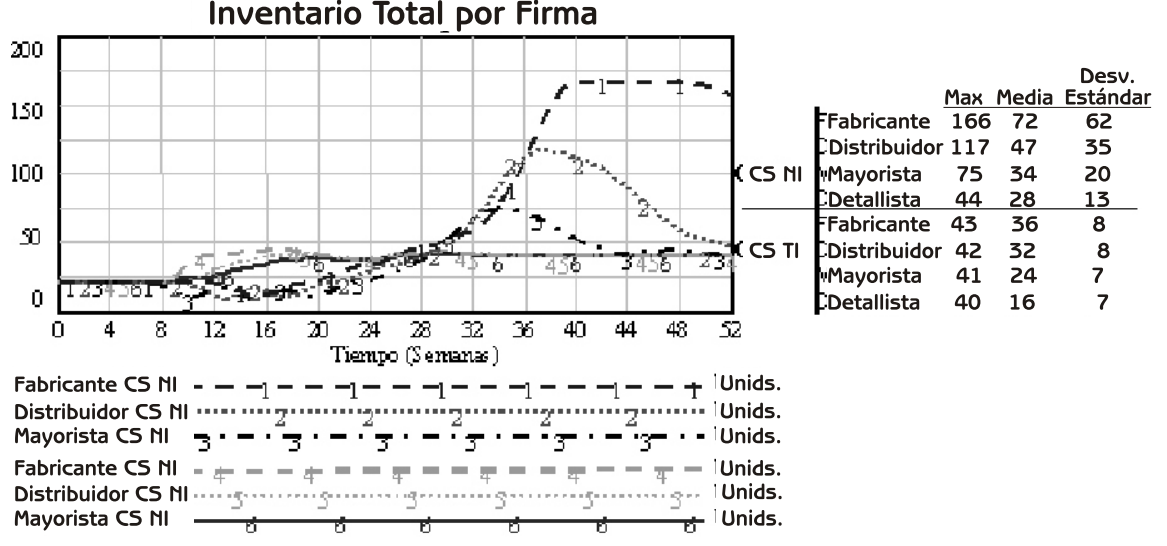

Figura 4. Resultados sobre el inventario total

unos niveles de inventarios más uniformes.

La primera línea de la tabla 4 muestra cómo la integración puede contribuir a lograr unas mayores ventas (throughput) (esta es la medida de las entregas acumuladas de los detallistas a los clientes finales en las 52 semanas). También se muestra en la tabla 4 que, la medida del nivel de servicio (porcentaje de órdenes recibidas de los clientes y satisfechas a tiempo), aumenta gradualmente a medida que mejora la colaboración entre las firmas. La tabla 4 revela también que la CS no integrada incrementa las necesidades de fondos con el tiempo, como una consecuencia, principalmente de las mayores necesidades de financiación de los inventarios, especialmente durante el incremento de la demanda. 
Mejora del Rendimiento Operativo y Financiero de las Cadenas de Suministro mediante el uso de Herramientas de Colaboración basadas en Internet. Un Enfoque Sistémico

\begin{tabular}{|c|c|c|c|}
\hline \multirow[b]{2}{*}{ Medida } & \multicolumn{3}{|c|}{ Nivel de Integración } \\
\hline & MI & PI & $\mathrm{TI}$ \\
\hline Producción acumulada (Unidades) & 372 & 393 & 393 \\
\hline Mivel de servicio (\%) & 73 & 90 & 96 \\
\hline Mec. Medidas de fondos normalizadas (\$M) & 1.26 & 1.09 & 1.0 \\
\hline
\end{tabular}

Tabla 4. Resultados de medidas operativas globales

\section{CONCLUSIONES}

Los resultados obtenidos muestran claramente las mejoras potenciales de la integración mediante el uso de herramientas de colaboración ofrecidas por las tecnologías de la comunicación en la cadena de suministro. Se alcanzan niveles de eficacia (entregas) y eficiencia (nivel de servicio) de la cadena de suministro notables, a su vez, se contrarresta significativamente el efecto bullwhip a lo largo de la cadena. Las herramientas de la colaboración basadas en Internet ofrecen la posibilidad de mejorar los procesos de gestión de las operaciones financieras a lo largo de la cadena, globales y locales. Operativamente la integración: (a) habilita las políticas de pedidos para ajustar las necesidades nuevas de los clientes anticipadamente, con mayor certeza, y con una gestión más eficiente de los inventarios y de los flujos de abastecimiento a lo largo de la cadena, (b) propicia menores necesidades relativas de fondos de operación y por supuesto menores costos financieros.

\section{BIBLIOGRAFÍA}

- BOWERSOX, D. J. 1997. Integrated Supply Chain Management: A Strategic Imperative, presented at the Council of Logistics Management 1997 Annual Conference, 5-8 Oct. Chicago, IL.

- CAVIMATO, J. L. 1992. A total Cost/Nalue Model for Supply Chain Competitiveness. Journal of Business Logistics, Vol. 13, Mo. 2, pp. 285-301.

- COOPER, M. C. AMD ELLRAM, L. M. Characteristics of Supply Chain Management and the Implications for Purchasing and Logistics Strategy. The International Journal of Logistics Management, 1993, Vol. 4, Mo. 2, pp. 13-24.

- CRISTOPHER, M. 1992. Logistics and supply chain management. London: Pitman Publishing.
- GILL, P. AMD J. ABEMD. 1997. Wal-Mart: The Supply Chain Heavyweight Champ. Supply Chain Management Review 1(1): 8-16.

- HEWITT F. 1994. Supply Chain Redesign. The International Journal of Logistics Management, Vol. 5, Mo. 2, pp. 1-9.

- LEE, H., and WHAMG, W. 1998. Information Sharing in a Supply Chain. Research paper Mo. 1549, Stanford University.

- MAKRIDAKIS, 5., 5. WHEELWRIGHT and R. HYMDMAM. 1998. Forecasting methods and Applications. John Wiley and Sons.

- MentZeR, J. T. 1993. Managing channel relations in the 21 st century. Journal of Business Logistics, Vol. 14, Mo. 1, PP. 27-42.

- POIRIER C.C. "Advanced Supply Chain Management". Berret-Koehler Publishers, Inc. San Francisco. 1999.

- RAGHumathaM, S. 1999. Interorganizational Collaborative Forecasting and Replenishment Systems and Supply Chain Implications. Decision Sciences 30(4): 1053-1071.

- SCOTT, C. AMD WESTBROOK, R. Mew Strategic Tools for Supply Chain Management. International Journal of Physical Distribution and Logistics Management, 1991, Vol. 21 Mo. 1, pp. 23-33.

STERMAM, J. 1984. Instructions for Running the

- Beer distribution Game (D-3679). Sloan School of Management, MIT.

- Stermar, J. D. 1989. Modeling Managerial Behavior: Misperceptions of Feedback in a Dynamic Decision Making Experiment. Management Science, Vol. 35, Mo. 3, March 1989.

- STEVens, G.C. 1989. Integrating the Supply Chain. International Journal of Physical Distribution \& Materials Management 19:3-8.

- TOWILL, D. R., Maim, M.M., and Wikner, J. 1992. Industrial Dynamics Simulation Models in the Design of Supply Chains. International Journal of Physical Distribution and Logistics Management 22:3-13.

- TVERSKY, A., KAHMEMAM D. 1974. "Judgement under Uncertainty. Heuristics and Biases". Science, 185, September. 1124-1131. 